\title{
Más confianza para una mejor escuela: el valor de las relaciones interpersonales entre profesores y director
}

\author{
More trust for a better school: the value of interpersonal relationships \\ between teachers and school principals
}

ISSN 1510-2432 - ISSN 1688-9304 (en línea) - DOI: https://doi.org/10.18861/cied.2017.8.1.2639

\begin{abstract}
Alicia Razeto
Doctora en Ciencias de la Educación, Pontificia Universidad Católica de Chile. Magíster en Gestión y Políticas Públicas, Universidad de Chile. Investigadora en trabajo social, sistema educativo y el estudio de estrategias para incrementar la relación entre familias y escuela. Académica, Escuela de Trabajo Social, Facultad de Ciencias Sociales, Pontificia Universidad Católica de Chile.
\end{abstract}

Fecha de recibido: 13/10/2016

Fecha de aceptado: 30/01/2017

\section{Resumen:}

Desde fines de la década de los noventa, investigadores del ámbito educacional han reconocido a la confianza interpersonal presente en las instituciones educativas como un recurso central para el mejoramiento educativo. Partiendo de esta premisa, el presente artículo tiene dos objetivos. El primero es identificar las ventajas que puede brindar a las organizaciones escolares la confianza interpersonal entre profesores y director. El segundo es describir cómo se presenta la confianza interpersonal en dos escuelas primarias chilenas. El primer objetivo se aborda a través de la revisión de los resultados de un conjunto de estudios publicados en contexto nacional e internacional. El segundo objetivo se trabaja a través de un estudio de caso aplicado en dos escuelas chilenas, utilizando las siguientes técnicas de recolección de información: un cuestionario a profesores, observaciones no participantes de reuniones de trabajo y entrevistas semiestructuradas a profesores y directores.

Los resultados muestran que la presencia de confianza en las relaciones interpersonales es ventajosa para las organizaciones escolares y que una de las fuerzas que la originan son los criterios de discernimiento que las personas utilizan para determinar cuán confiable es el otro. Los resultados del estudio establecen diferencias entre las escuelas: en una de ellas hay altos niveles de desconfianza y en la otra hay mayor presencia de confianza en las relaciones interpersonales entre profesores y director.

Palabras clave: confianza, relaciones interpersonales, mejoramiento educativo

\begin{abstract}
:
As from the late nineties, researchers in the educational area have recognized the interpersonal trust at schools as a central resource for the educational improvement. Based on this premise, this article has two aims. The first one is to identify the benefits that the interpersonal trust between teachers and the principal or headmaster can offer to the school organizations. The second one is to describe how we approach the interpersonal trust in two elementary Chilean schools. The first aim is analyzed across the review of results of a set of studies published in national and international context. The second aim is handled through the accomplishments of a case study in two Chilean schools using as information gathering strategies: a questionnaire to teachers, not participating observations at work meetings and semi-structured interviews to teachers and the principals. The results
\end{abstract}


show that the presence of trust in the interpersonal relations is beneficial for the school organizations, and that one of the forces that creates trust are the discernment criteria used by the people to determine how reliable the other one is. The results of the case study establish differences among the schools, in one of them there are high levels of distrust and in the other one a greater presence of trust was found in the interpersonal relations between teachers and the principal.

Keywords: trust, interpersonal relationship, school improvement

\section{Introduccion}

La confianza es un valor social que puede manifestarse de tres formas interrelacionadas (Misztal, 1996):

1. Como propiedad de los individuos, es decir, como característica de la personalidad.

2. Como propiedad de las relaciones interpersonales, atributo colectivo que permite alcanzar ciertas metas grupales u organizacionales. A esta manifestación de la confianza se le conoce como confianza interpersonal.

3. Como propiedad del sistema social y valor público. A esta forma se le llama confianza social.

El presente artículo asume la comprensión de la confianza como una cualidad de las relaciones interpersonales (Sztompka, 1999). La confianza es un concepto y proceso relacional en el que coexisten tres elementos: alguien que confía, alguien en quien se confía y aquello que se confía (Parra, 2001). Una relación de confianza se caracteriza principalmente por dos aspectos: i) La existencia de expectativas en el comportamiento de los demás por parte de quien confía, y; ii) La aceptación por parte de quien confía, de la condición de ser vulnerable frente al otro (Barber, 1983; Mayer y Schoorman, 1995; Misztal, 1996; Luhmann, 1996; Hardin, 2002; Cook et al., 2005).

En el caso de la desconfianza, ésta se define como las expectativas negativas sobre la conducta de los demás vinculadas a la creencia de que el otro tiene intenciones dañinas, lo que trae aparejado el consecuente deseo de protegerse de los efectos de su conducta, entendida no sólo como acciones sino también como palabras e intenciones (Lewicki et al., 1998).

Asumiendo la confianza interpersonal como constructo fundamental, este artículo presenta dos propósitos. El primero es analizar la relación que existe entre la presencia de la confianza en profesores y directores y la efectividad de las escuelas. Este primer propósito se trabaja a partir de una revisión de estudios empíricos realizados a nivel nacional e internacional en el campo de las ciencias sociales y la educación. En tanto, el segundo propósito es describir el estado de las relaciones interpersonales -y de la confianza interpersonal- en dos escuelas básicas o primarias chilenas. Este propósito se desarrolla en base a la realización de un estudio de caso que se efectuó en el año 2014. De este modo, las preguntas que el artículo responderá son: ¿Cuáles son las ventajas que representa para una escuela la presencia de confianza interpersonal entre profesores y director? y ¿Cómo se presenta la confianza interpersonal en dos escuelas chilenas básicas?

\section{Más confianza contribuye a una mejor escuela. ¿qué dicen los estudios?}

La confianza ha sido estudiada desde distintas disciplinas (Misztal, 1996), entre las que destacan la psicología, la sociología, las ciencias políticas y la antropología (Shalins, 1972). El tema ha dado origen a numerosos estudios que enfatizan una mirada particular sobre la confianza de acuerdo a si es concebida como característica de la personalidad (Deutsch, 1958; Cole, 1973; Wolfe, 1976) de las relaciones interpersonales o de la sociedad. 
Desde la sociología y las ciencias políticas la confianza se ha estudiado mayormente a través de la teoría del capital social. Un resultado común de los estudios es que conciben a la confianza como poseedora de atributos virtuosos que generan positivos efectos en las personas, organizaciones y sociedades. Desde la teoría del capital social Coleman (1998) señala que un grupo que tiene una alta confiabilidad y confianza generalizada es capaz de lograr mucho más que un grupo comparable sin dicha confianza. También desde dicha teoría, Putnam $(1995,2000)$ plantea que la confianza y las redes facilitan la coordinación y comunicación, promoviendo la generación de sentido de nosotros y generando beneficios por la acción colectiva. La confianza hace más eficiente a una sociedad, mucho más que a una que esté marcada por la desconfianza. Dice Putnam (1995) que la confianza lubrica la vida social, y que la interacción frecuente entre diversos conjuntos de personas puede facilitar la cooperación y el beneficio mutuo.

Por su parte, el politólogo estadounidense de origen japonés Francis Fukuyama (1996) asigna al capital social una responsabilidad importante en el logro del desarrollo económico y político. Este tipo de capital es la capacidad que nace a partir del predominio de la confianza en una sociedad o en determinados sectores de la misma (Fukuyama, 1996). Sería decisivo para el éxito de la democracia y sería un componente vital para el desarrollo económico, ya que "una de las lecciones más importantes que podemos aprender del análisis de la vida económica es que el bienestar de una nación, así como su capacidad para competir, se halla condicionado por una única y penetrante característica cultural: el nivel de confianza inherente a esa sociedad" (Fukuyama, 1996). Dice Fukuyama que la confianza genera organizaciones más eficientes donde no se requieren extensos contratos ni tampoco una regulación legal porque el consenso moral previo provee a los miembros del grupo una base de confianza mutua.

Por una parte, la confianza desempeña un papel relevante en la efectividad de las organizaciones, facilitando la cooperación entre individuos y grupos, promoviendo la aceptación de relaciones solidarias y contribuyendo a generar una comunidad más inclusiva, integradora y cosmopolita (Misztal, 1996). Desde los estudios organizacionales y de la administración se admite que la confianza trae muchos beneficios, aumentando los resultados que las personas son capaces de alcanzar (Kramer, 1999). Cuando en una empresa sus miembros tienen confianza, aumenta la rapidez y disminuyen los costos, lo que tiene incidencia en la productividad de la organización (Covey y Merrill, 2007). Por otra parte, la confianza traería impactos positivos en la comunicación, coordinación y generación de acciones en equipo para el logro de las metas formuladas en una organización (Kramer y Tyler, 1996; Wicks, Berman y Jones, 1999; Gillespie y Mann, 2000; Ferres y Travaglione, 2003; Saunders y Thornhill, 2004; Harjinder, et al., 2005; Dar, 2010).

Las ventajas que la confianza trae para las organizaciones también alcanzan a las organizaciones escolares debido a que éstas basan su acción en las relaciones interpersonales a partir de los distintos roles que asumen sus miembros. El efecto de la confianza en la productividad de las organizaciones escolares ha sido "...razonable y empíricamente apoyada en la literatura escolar" (Forsyth, Barnes y Adams, 2006). En los Estados Unidos de América se han llevado a cabo numerosos estudios que mencionan la asociación entre confianza y efectividad de la escuela (Bryk \& Schneider, 2002; Uline, Miller y Tschannen-Moran, 1998; Hoy y Henderson, 1983; Forsyth, Barnes y Adams, 2006; Goddard, Tschannen-Moran y Hoy, 2001; Tschannen-Moran y Hoy, 2000; Park, Henkin y Egley, 2005; Hoy y Tschannen-Moran, 1999; Uline et al., 1998). Por ejemplo, el estudio de Forsyth, Barnes y Adams (2006) concluye que la confianza entre profesores y directores influye en la producción de tres resultados educativos: 
1. Mayor sentido de eficacia colectiva de los profesores, pues si hay una historia basada en un comportamiento consistente y expectativas positivas, los profesores creerán en su eficacia.

2. Menor estructura burocrática, ya que la confianza puede reducir la necesidad de mecanismos burocráticos de control y evitar la proliferación de reglas y supervisión cercana.

3. Mejor desempeño académico de profesores y estudiantes, ya que la confianza funciona como lubricante para la cooperación, aumentando la productividad.

En el contexto educativo, las relaciones interpersonales basadas en la confianza incrementan la eficiencia en el proceso de formación (Zapata et al., 2010). La confianza se considera una propiedad de las organizaciones escolares que constituye un recurso central para el mejoramiento educativo (Bryk y Schneider, 2002). Estos autores proponen el concepto de confianza relacional, el que definen como aquel tipo de confianza que se genera a partir de la sincronía entre las expectativas y el cumplimiento de las obligaciones de cada actor de la organización. En este plano, los líderes educativos asumen un papel muy importante, sobre todo en instituciones vulnerables desde el punto de vista social en las que las personas (estudiantes y profesores) pasan a ser el motor de la gestión directiva (Tapia et al., 2011).

Según Bryk y Schneider (2002) las relaciones interpersonales que se desarrollan entre director, profesores, estudiantes, padres y apoderados pueden ser recursos de mejoramiento y progreso para las escuelas. Los autores plantean que "la naturaleza del intercambio social y los rasgos de la cultura local condicionan la capacidad de la escuela para mejorar" (Bryk y Schneider, 2002). Particularmente, la confianza presente en las relaciones interpersonales facilita el funcionamiento diario de la escuela, actuando como un recurso crítico para que los líderes generen un ambiente con estrategias de mejoramiento. La confianza puede asumir tres formas: confianza orgánica, confianza contractual y confianza relacional (Bryk y Schneider, 2002). La forma que asume la confianza dependería de la naturaleza de la institución social en la cual se producen las relaciones interpersonales.

Bryk y Schneider (2002) postulan que la confianza contractual no es suficiente para el buen funcionamiento de las organizaciones escolares. Si bien es importante que las escuelas cuenten con una clara base contractual, dada la naturaleza social y moral del quehacer educativo también es necesario actuar de acuerdo a ciertas motivaciones comunes, tomar decisiones de forma colectiva, generar esfuerzos extracontractuales para lograr los objetivos y movilizar la interdependencia entre los profesores y directivos hacia el logro de las metas de la organización escolar. De esta forma, Bryk y Schneider construyen el concepto de confianza relacional para enfatizar los fundamentos ético-sociales que están en la base del funcionamiento de las escuelas.

Según Bryk y Schneider (2002) la confianza relacional cataliza consecuencias clave para la organización escolar como lo son, por un lado, crear una cultura de escuela de apoyo que realce el compromiso con esta y genere una orientación positiva hacia el cambio y, por otro lado, facilitar la estructura de trabajo para la toma de decisiones en el conjunto de la comunidad de profesionales, con rendición de cuentas y apoyo para la enseñanza del profesor. Esto influiría en el ámbito instruccional mejorando el compromiso y aprendizaje de los estudiantes. Las consecuencias clave de la confianza retroalimentan la expansión de la base de la confianza.

Una organización que tiene confianza relacional va a tender a la reproducción y 
profundización de este tipo de relaciones entre los profesores, directivos y comunidad educativa en general. Ello debido a que fomenta una cultura de trabajo de apoyo en la que existe compromiso con la escuela y en la que se impulsa una positiva orientación hacia los cambios. Asimismo se promueve una mejor estructura de trabajo en la que se facilita la toma de decisiones colectivas en base a la rendición de cuentas.

Una de las fuerzas modeladoras de la confianza la constituye la forma en la que operan un conjunto de criterios que las personas utilizan para discernir acerca de cuán confiable es el otro. Bryk y Schneider (2002) plantean que existen cuatro criterios de discernimiento de la confianza que sirven como lentes para observar e interpretar las intenciones de otros en el contexto escolar. La aplicación de estos criterios resulta en un juicio acerca de la confiabilidad. Estos son:

- Respeto. Se refiere al reconocimiento de la importancia del rol que cada persona tiene en la educación de los niños y la mutua dependencia que existe entre las partes involucradas en esta actividad. El respeto necesita ser recíproco. Lipman (1990) plantea que hay dos formas de respeto: una basada en lo que la otra persona ha hecho y, la otra, basada en la posición o estatus.

- Competencia. Se refiere a la ejecución de las responsabilidades formales de los individuos. Tiene que ver con la habilidad que cada uno demuestra para lograr los resultados deseados.

- Estima personal por los otros. Su sentido es el sentirse cuidado por el otro, entendiendo que cualquier acción de los miembros para reducir el sentimiento de vulnerabilidad influye positivamente en la confianza interpersonal.

- Integridad. En un sentido básico, tiene que ver con la consistencia entre lo que se dice y se hace. En un sentido más profundo, abarca la perspectiva ético-moral que guía el trabajo de una persona.

Según Bryk y Schneider (2002) este nivel de las relaciones es determinante en la formación de las relaciones de rol y en la producción de confianza a nivel organizacional, la que conducirá a sincronizar las expectativas con las obligaciones de cada miembro de la comunidad educativa, aspecto central de la confianza relacional.

\section{¿Cómo es la confianza interpersonal en una escuela chilena? estudio de caso}

\section{Método}

El estudio de caso fue llevado a cabo en dos escuelas básicas o primarias de la capital de Chile (Santiago): una pública o municipal y una privada que recibe aportes monetarios del Estado. La investigación adoptó un enfoque metodológico cualitativo y de nivel exploratorio. Las técnicas de investigación aplicadas fueron las siguientes: un cuestionario a profesores, observaciones no participantes de reuniones de trabajo y entrevistas semiestructuradas a profesores y directores.

El cuestionario se utilizó para analizar las relaciones de confianza. Fue adaptado del cuestionario creado por Hoy y Tschannen-Moran (1999), disponible en el sitio Web de la investigadora Tschannen-Moran ${ }^{1}$. De acuerdo con estas investigadoras, el instrumento permite crear un índice que ayuda a comprender las relaciones al interior de la escuela y la presencia de confianza en ellas. El cuestionario fue respondido por un total de 30 profesores, 19 de la escuela pública y 11 de la escuela particular. El Índice de confianza agrupa el puntaje obtenido por tres subescalas (confianza en el director, en colegas y en estudiantes y familias). 
La observación se focalizó en las relaciones interpersonales asociadas al trabajo cotidiano de profesores y director. Se observó un total de 25 sesiones de reuniones técnicas en ambos establecimientos. Las reuniones técnicas consisten en una instancia de encuentro periódico en la que participan profesores y director. En ellas se tratan distintos asuntos de interés para la escuelas tales como planificación de actividades, evaluación de alumnos, preparación de ceremonias, preparación de reunión de apoderados y talleres, entre otros. La entrevista fue la técnica principal en la etapa de recolección de información. A través de dichas entrevistas se logró detectar cómo se presentaba la confianza interpersonal entre los miembros de la comunidad educativa. En las entrevistas participaron un total de 22 profesores, 2 directores y 2 jefes de la Unidad Técnico Pedagógica (UTP). El jefe de UTP es un profesional que, entre otras labores, es responsable del asesoramiento al director y de la programación, organización, supervisión y evaluación del desarrollo de las actividades curriculares (Fundación Chile, 2006).

\section{Resultados del estudio de caso}

\section{Índice de confianza}

A partir de los resultados del cuestionario se construyó un Índice de confianza para las escuelas. El mismo fue elaborado en base a tres referentes:

Confianza de los profesores en el director

Confianza de los profesores en sus colegas

Confianza de los profesores en los estudiantes y familias ${ }^{2}$

Aplicando los procedimientos de cálculo propuestos por Tschannen-Moran y Hoy se llegó a un puntaje del total de subescalas para cada escuela, lo que permitió comparar sus realidades. Los procedimientos de cálculos y fórmulas utilizados fueron los sugeridos por Tschannen-Moran y Hoy en los documentos Directions for Administering Trust Surveys y Scoring Directions for Faculty Survey ${ }^{3}$.

Considerando los pasos previamente descritos, el índice para cada escuela resultó ser el siguiente (Tabla 1):

Tabla 1: Puntajes estandarizados de las escuelas de acuerdo a subescalas

\begin{tabular}{|l|c|c|}
\hline \multicolumn{1}{|c|}{ Subescalas } & Puntaje escuela particular & Puntaje escuela pública \\
\hline $\begin{array}{l}\text { Subescala confianza de } \\
\text { profesores en director }\end{array}$ & 362 & 474 \\
\hline $\begin{array}{l}\text { Subescala confianza de } \\
\text { profesores en colegas }\end{array}$ & 130 & 358 \\
\hline $\begin{array}{l}\text { Subescala confianza de } \\
\text { profesores en padres y } \\
\text { estudiantes }\end{array}$ & 341 & 395 \\
\hline
\end{tabular}


A partir de los puntajes es posible concluir que:

- La confianza de los profesores en el director fue el referente que tuvo mejor puntaje (362) en la escuela particular, seguida de la confianza de los profesores en sus estudiantes y padres (341) y, por debajo, la confianza de los profesores en sus colegas (130).

- La confianza de los profesores en el director fue el referente que tuvo mejor puntaje (474) en la escuela pública, seguida de la confianza de los profesores en sus estudiantes y padres (395), y un poco más abajo la confianza de los profesores en sus colegas (358). Todos los puntajes superan los de la escuela particular.

- En las tres subescalas, ambas escuelas presentan puntajes bajo el promedio de la muestra estadounidense en la cual se basaron las normas de cálculo (500 puntos).

La escuela particular obtuvo bajos puntajes de acuerdo al rango de puntajes estandarizados, por debajo del promedio del $84 \%$ de las escuelas de la muestra del estudio norteamericano. Para el caso de la confianza de profesores en colegas, la escuela está por debajo del 99\% del promedio de la muestra. En la escuela pública, la subescala de la confianza de profesores en el director se encuentra casi en el promedio de la muestra estadounidense. Las demás subescalas están por debajo del promedio del $84 \%$ de las escuelas de la muestra del estudio norteamericano.

\section{Criterios de discernimiento para juzgar la confiabilidad}

Una fuerza fundamental en la construcción de la confianza interpersonal es la forma en la que operan los criterios de discernimiento. A través de las entrevistas y observaciones, la investigación logró detectar la forma en la que operan estos criterios y su contribución a la formación de confianza interpersonal.

\section{Respeto}

Compartir la misma profesión y posición fue la principal fuente de respeto entre los profesores de ambas escuelas, sentimiento acentuado en los profesores del colegio público al ejercer su rol en un contexto social desfavorable del estudiantado. Este aspecto compartido por ellos les hacía ser conscientes de la importancia de su rol dentro de la organización escolar.

No ocurrió de la misma manera en otro plano relacional, el de los profesores con el jefe de la UTP. Tanto las entrevistas como las observaciones realizadas constataron una situación similar en ambos establecimientos: un respeto altamente susceptible de ser vulnerado por desacuerdos y conflictos originados por el trabajo en la escuela debido al aumento de las exigencias con motivo de la implementación de la nueva política educativa a favor de la calidad escolar4.

En el caso de la escuela pública, a esta situación se le sumaban las críticas del profesorado al jefe de UTP por su relativa ausencia en la organización, pues a pesar de tener una jornada de trabajo por el máximo legal, se desempeña en otros dos establecimientos además de sus actividades en la municipalidad. Esta situación resta cantidad y calidad a las interacciones, lo que debilita la comunicación y afecta el respeto en el sentido de valorar al otro en el rol que asume en la escuela, escuchar sus preocupaciones e incorporarlas en la gestión de la escuela (Bryk y Schneider, 2002). 
El aspecto de mayor diferencia entre una y otra escuela estuvo representado por el respeto de los profesores hacia la directora. En el caso de la escuela privada, se encontró que el respeto estaba basado más en la posición o estatus de la directora -por ser la dueña del colegio desde hace más de 20 años- que en los resultados de sus acciones. En este caso, la posición y concentración de poder que detenta la directora influyen en la actitud de respeto que los profesores demuestran hacia sus órdenes y propuestas. Por ejemplo, ella puede tomar la decisión de desvinculación laboral, como se aprecia en esta opinión de una de las docentes de la escuela:

“Es que cuesta confiar en la gente. En un colegio particular subvencionado la situación es diferente, no es como una escuela municipal, es diferente el sistema, entonces cuesta... Es que aquí uno, en este caso, está trabajando directamente con el dueño, en cambio allá en el municipal estamos solos, está la directora y todo lo demás, pero no está el dueño encima de uno. Aquí uno está... allá no... en un colegio municipal hay un sumario y después viene todo un... si uno comete errores o una falta...no la despiden así nomás, le hacen un sumario. En cambio acá no po, se termina el contrato y se va, es diferente la cosa..." (Profesora 10, Escuela Particular Subvencionada)

Muy distinto era lo que ocurría en la escuela pública, en la cual el respeto fue bien evaluado en la relación entre docentes y la directora. En este caso, el respeto está basado tanto en su autoridad como en los hechos (Lipman, 1990), ya que la mayoría de los docentes concuerda en reconocer son capacidades de liderazgo y su compromiso.

\section{Competencia}

Uno de los primeros aspectos que llama la atención en el criterio de competencia es que, en ambas escuelas, tanto profesores como directivos tienden a asociarlo con las ideas de compromiso, vocación y responsabilidad más que con la habilidad para lograr los resultados esperados, que es como lo definen Bryk y Schneider (2002). Tal vez esto pueda relacionarse con el hecho de que ambos establecimientos han obtenido resultados en el Sistema de Medición de la Calidad de la Enseñanza por debajo del promedio nacional. Sin embargo, en la escuela particular se halla un discurso más crítico. De hecho, los profesores cuestionan la competencia de la directora por su desactualización y falta de capacitación, tal como se aprecia en la siguiente cita:

“Pero la Sra. X no sé (si confío en su capacidad de trabajo), porque ella hace tanto tiempo que dejó de hacer clases que de repente podría dar su opinión pero ya no tiene los conocimientos muy claros de ahora, cómo se hacen las clases, qué tipo de metodología o estrategia poder usar adentro de la sala, uno va quedando en el pasado..." (Profesora 5, Escuela Particular)

En cambio, en la escuela pública la competencia profesional fue evaluada positivamente entre profesores y la directora. Reconocen en ella muchas capacidades y compromiso por el progreso de la organización. La opinión de ellos cambia al momento de referirse al jefe de UTP quien, a pesar de ser considerado un hombre capaz, no logra ejercer eficazmente su rol, tal como vemos en el siguiente extracto de entrevista: 
"a ver, tenemos un jefe de UTP que tiene una labia hermosa, sabe mucho, pero le falta concretar, ¿ya? Le falta concretar, concretar cosas. Yo insisto, yo estoy estudiando y yo puedo ver parámetros, he tenido que ir a colegios de otros colegas, ¿ya?, y he visto el manejo que tienen otros jefes de Unidad Técnica Pedagógica, referente a éste, a mí me hace hacer muchas cosas, me exige y yo cumplo, ¿ya?, pero faltan elementos que tienen que llenar, porque yo no saco nada conque otra persona me revise el libro y me ponga timbre, ¿de qué sirve?, ¿de qué sirve si yo puedo poner cualquier cosa ahí? No sirve de nada..." (Profesora 6, Escuela Municipal).

\section{Estima personal hacia los demás}

Según Bryk y Schneider este criterio es uno de los más poderosos en la creación de confianza interpersonal dada la naturaleza relacional del quehacer educativo. Las señales de estima o aprecio gatillan esfuerzos más allá de lo establecido por el rol formal pues incrementan el sentimiento de integración social, el significado y el valor como profesionales (Bryk y Schneider, 2002).

Pese a la importancia de este criterio, en la escuela particular se encontraron importantes problemas en la relación entre docentes y el jefe de la UTP. Uno de los factores que influyeron notablemente en la aparición de problemas en relación a las señales de estima o aprecio fue el hecho de que en la escuela trabajan varios (7) familiares de la directora. Para el grupo de profesores que no tiene relaciones de parentesco con la directora esta situación los hace sentirse en una posición desventajosa dado que asumen la creencia de que los familiares tienen ciertos privilegios. Además estos actuarían como aliados de los intereses de la directora con la consecuente acentuación del sentimiento de vulnerabilidad e incertidumbre en la que los docentes desempeñan sus labores, lo que genera un clima donde no se construye aprecio por las personas ni se potencia su valor como profesionales, tal como se detecta a través del siguiente registro de observación.

“(...) Cuando no confían en ti uno empieza a hacer todo mal".

Cuenta que la grabaron con una cámara, el hijo de la directora, la instalaron en un esqueleto y la grabaron sin avisarle. Le sucedió a ella y a dos profesoras más. Dice: “uno se siente pasado a llevar". Otra profesora la apoya: "ese es un tema, las confianzas; hay que tratar de que el otro tenga confianza en ti"; "que llevando tanto tiempo todavía no me tengan confianza; eso me molesta". Esto sucedió en octubre. ..." (Registro de observación, 2 de dic. 2010, escuela particular).

El problema con el criterio del respeto generó consecuencias en el plano de la estima personal hacia los demás. Esto fue lo que ocurrió en el caso de la escuela privada, en la relación entre un grupo de docentes y el jefe de la UTP. Los profesores asumen que este profesional tiene intenciones dañinas debido a que le atribuyen la responsabilidad del aumento de la exigencia en el trabajo a propósito de la nueva política escolar, lo que provoca sobrecarga y agotamiento.

“... él asumió el puesto y empezó a criticarnos. Una vez dijo que nuestro trabajo era paupérrimo, y yo le dije, "¿y con qué cara dice usted esto?, si usted estuvo un año con licencia. (...) Entonces ahora esta persona, que no tiene el título de jefe de UTP, que venga a decirnos a uno a la pinta de él como se le ocurre la cosa, entonces ahí empiezo a tener problemas con él. Los demás, le dije yo, los demás tú podrás cuentiarlos P. y todo, pero a mí no me vení a cuentiar, tú nos inventai trabajo" (Profesor 9, Escuela Particular) 
En el colegio privado hay un grupo de profesores que asocia positivamente la estima personal con la figura de la directora gracias a la concesión de permisos especiales por parte de ésta para ausentarse o llegar más tarde al establecimiento por problemas personales. Eso lo consideran como una señal de aprecio que genera estima hacia ella por parte de los docentes.

En el colegio público, una actividad que ha sido fuente de integración y aumento de la estima personal hacia los demás es el compartir un desayuno los días viernes en la mañana. Adicionalmente, los profesores expresan aprecio y afecto por la directora, los que obedecen al compromiso y dedicación al progreso de la organización, a su preocupación por las oportunidades de capacitación, al apoyo a la gestión de los docentes y a su manifestación permanente de preocupación por la vida personal de los profesores. Esto último es destacado tanto por un grupo de docentes -quienes agradecen su capacidad de escucha y apoyo cuando atraviesan por dificultades personales-como por la propia directora, quien asegura conocer la vida personal de la mayoría de los profesores de la escuela, como se observa en la siguiente cita de entrevista:

"Yo con todos converso harto así a puertas cerradas. Conozco todas sus vidas, ¿me entiende? La que tiene problemas, con la mamá, yo sé cómo está la mamá, la edad que tiene, cuántos hijos, las características del yerno, etcétera. Ese tipo de cosas, les conozco la vida a todos" (Directora, Escuela Pública).

\section{Integridad}

Así como ocurre con el criterio anterior, la integridad es un componente que tiene mucha fuerza en la creación de confianza interpersonal. Según Bryky Schneider (2002) la integridad refleja los principios ético-morales de los otros pues tiene que ver con el tránsito desde los objetivos individuales de cada miembro de la comunidad escolar a los objetivos colectivos de la organización. Produce compromiso entre los miembros de la organización generando actitudes de cooperación y solidaridad para lograr las metas comunes, lo que implica actuar velando por los intereses de los niños.

En el colegio privado, este criterio fue mal evaluado en todos los planos de las relaciones: entre profesores y entre docentes, jefe de UTP y directora. La asociación del colegio con el interés de lucro fue lo que más afectó las opiniones en torno a la integridad. Esto se debe a la naturaleza particular de la organización pero, por sobre todo, a la duplicidad de roles de quien la dirige, como directora y sostenedora. Es importante señalar que en Chile, el rol de sostenedor de un establecimiento educacional corresponde al dueño de un colegio particular. Ante esta circunstancia, los profesores infieren que la directora -y los empleados de su confianza- preferirá velar por sus intereses personales (el progreso de su negocio) por sobre los intereses de los docentes (que son sus condiciones de trabajo), tal como se aprecia en el siguiente extracto:

“Es que también ella está en una posición de jefe, ella es la dueña, ella tiene que velar por sus intereses entonces ella igual tiene que tener un perfil de defender su empresa, o sea, no es que ella vaya a dejar que hagan lo que quieran los trabajadores" (Profesor 2, Escuela Privada).

Otra causa de problemas con la integridad lo reviste la traición a la confidencialidad de las conversaciones sostenidas entre profesores y directivos. Este problema también apareció en el colegio público, como se aprecia en el discurso de esta profesora al referirse a su relación con el jefe de UTP: 
“Con él específicamente no, no confiaría en contarle algo porque yo sé que diría... y no es confiable. No es confiable, la directora tuvo una cosa la semana pasada y que no se lo dijera a nadie, y yo confío en usted, dígaselo, yo se lo digo a usted. Entonces uno ve al tiro qué poca confianza. Entonces él con toda la gente es así..." (Profesora 9, Escuela Municipal).

La discreción y el respeto a la confidencialidad son aspectos muy valorados por los profesores para atribuir confianza a otro, sobre todo con respecto a alguien que ejerce un cargo directivo en el colegio.

La falta de discreción en las comunicaciones disminuye fuertemente la confiabilidad en el otro pues reduce la probabilidad de comunicaciones futuras, tal como se advierte en la siguiente opinión de una de las profesoras del colegio privado:

“Ahí si que hay que tener cuidado porque por ejemplo en P. no se puede confiar, en P.
sí que no se puede confiar, porque yo le puedo estar diciendo ‘sabe que la directora tal
cosa', o ‘a mí me gustaría que esto fuera así así' y él va y acusa, o le dice a la directora
para que llame la atención o si no hemos cumplido con nada él nos acusa. O lo otro que
también tiene el P., por ejemplo, si yo hice algo incorrecto o sea, un papel que esté mal
hecho, una planificación, se lo comenta a otra persona 'pero mira Z. cómo hace esto',
'mira F. cómo hace esto', entonces no es de confiar..." (Profesora 3, Escuela Particular).

En el colegio privado también existe un grupo de profesores que cuestiona la integridad de sus colegas por considerar que desempeñan su labor docente velando por sus propios intereses materiales y no por el bienestar de los niños. Bastante distinta es la situación de este criterio en el colegio público. Los docentes tienen una positiva evaluación de la integridad de la directora por el constante esfuerzo que realiza para lograr una buena marcha del colegio y por la dedicación diaria hacia el bienestar de los niños.

Otra de las prácticas que los docentes valoran de la directora es su responsabilidad, cumplimiento de compromisos, su discreción y fiabilidad. Consideran a la directora como una persona discreta y fiable, que los conduce incluso a comunicarle problemas de la vida personal sin temer que estos se vuelvan asuntos de conocimiento público.

Al contrario de lo que ocurre con la directora, los profesores cuestionan la integridad del jefe de UTP pues estiman que hay una brecha entre su discurso y sus acciones debido a que hay carencias en los resultados de su rol en la escuela, lo que genera una brecha entre los intereses personales de la autoridad y los de la escuela. Con respecto a la integridad entre profesores, las únicas fuentes de críticas la constituyen dos profesores cuya conducta es cuestionada del punto de vista ético por parte de sus colegas.

A modo de síntesis, la Tabla 2 muestra los criterios de discernimiento asociados a las principales características de las organizaciones escolares participantes del estudio, indicando si estas influyen positivamente o no en la confianza interpersonal. 
Tabla 2. Características organizacionales que influyen en la confianza interpersonal y criterios de discernimiento implicados

\begin{tabular}{|c|c|c|}
\hline $\begin{array}{l}\text { Escuelas } \\
\text { participantes } \\
\text { del estudio }\end{array}$ & $\begin{array}{l}\text { Características organizacionales que } \\
\text { influyen en la confianza relacional } \\
\text { según entrevistas y observaciones } \\
\text { realizadas }\end{array}$ & $\begin{array}{l}\text { Criterios de discernimiento } \\
\text { implicados }\end{array}$ \\
\hline \multirow[t]{3}{*}{ Escuela privada } & $\begin{array}{l}\text { Superposición de cargos de directora } \\
\text { y sostenedora del establecimiento (-) }\end{array}$ & $\begin{array}{l}\text { Integridad } \\
\text { Competencia } \\
\end{array}$ \\
\hline & Intereses de lucro personal (-) & $\begin{array}{l}\text { Integridad } \\
\text { Estima personal } \\
\text { Respeto } \\
\text { Competencia } \\
\end{array}$ \\
\hline & $\begin{array}{l}\text { Incorporación de familiares de la } \\
\text { directora (-) }\end{array}$ & $\begin{array}{l}\text { Competencia } \\
\text { Respeto } \\
\text { Estima personal } \\
\text { Integridad }\end{array}$ \\
\hline \multirow[t]{4}{*}{ Escuela pública } & $\begin{array}{l}\text { Existencia de un sostenedor municipal } \\
(+)\end{array}$ & $\begin{array}{l}\text { Competencia } \\
\text { Integridad } \\
\end{array}$ \\
\hline & $\begin{array}{l}\text { Falta de regulación del cumplimiento } \\
\text { de la jornada laboral (-) }\end{array}$ & $\begin{array}{l}\text { Competencia } \\
\text { Estima personal } \\
\text { Integridad } \\
\end{array}$ \\
\hline & $\begin{array}{l}\text { Escasez de incentivos y sanciones al } \\
\text { desempeño (-) }\end{array}$ & Competencia \\
\hline & $\begin{array}{l}\text { Alineamiento con conceptos de } \\
\text { políticas educacionales (+) }\end{array}$ & $\begin{array}{l}\text { Integridad } \\
\text { Competencia }\end{array}$ \\
\hline
\end{tabular}

(-) Indica una influencia negativa en los criterios de discernimiento de la confianza.

(+) Indica una influencia positiva en los criterios de discernimiento de la confianza.

En la escuela particular, la superposición de cargos de directora y sostenedora del establecimiento, el interés de lucro personal y la incorporación de familiares de la directora influyen negativamente en la confianza relacional en los criterios mencionados en la Tabla 2. En la escuela pública, la existencia de un sostenedor municipal y el alineamiento con conceptos de las políticas educacionales influyen positivamente en la confianza relacional. Sin embargo, la falta de regulación del cumplimiento de la jornada laboral y la escasez de incentivos y sanciones al desempeño lo hacen negativamente. 


\section{Conclusiones}

Las relaciones interpersonales son pilares fundamentales que sustentan el quehacer diario de las escuelas. Cuando están alimentadas por la confianza, las relaciones interpersonales entre profesores y directivos se constituyen en parte de los cimientos de la organización escolar que posibilitan el trabajo colectivo (Bryk y Schneider, 2002) y facilitan los procesos de mejora del funcionamiento para desarrollar un proceso sostenible de cambio asentado en el vínculo social.

Por un lado el presente artículo ha proporcionado una reflexión conceptual y descriptiva para respaldar la afirmación de que más confianza en las relaciones interpersonales entre profesores y directores puede generar las condiciones para una escuela más efectiva. Desde la revisión de los estudios sobre el tema se puede concluir que la confianza interpersonal genera efectos positivos en las organizaciones escolares y que, por lo tanto, vale la pena efectuar más estudios que respalden esta asociación en contexto chileno y latinoamericano. También es necesario considerar con mayor prioridad programas que incentiven y protejan espacios de convivencia interna entre los profesionales de los establecimientos escolares. Por otro lado, el estudio de caso permitió presentar formas de abordaje metodológico para analizar el complejo fenómeno de la confianza, las que podrían replicarse en otros estudios futuros. Asimismo permitió constatar que al menos en el contexto de las dos escuelas estudiadas la confianza es un fenómeno de alta complejidad que se presenta de manera muy distinta en cada organización y que tiene diversos obstáculos que provienen de la forma en la que cada persona juzga las intenciones de otros a través de los criterios de discernimiento para la confianza.

El estudio de caso concluye que los criterios de discernimiento utilizados para juzgar las intenciones de otros se encuentran profundamente relacionados entre sí al momento de confiar en los demás. Esta interrelación se expresa en que, por ejemplo, una de las posibles fuentes de respeto entre las personas es la competencia profesional, lo que fue demostrado positivamente en el caso de la directora del establecimiento público y negativamente en el caso de la directora y sus hijos en el establecimiento particular. A su vez, una relación que se sostiene en el respeto genera mayores posibilidades de producir estima personal entre las partes puesto que sentirán una intención de cuidado y de minimización de la vulnerabilidad propia de la labor docente. Es el caso de lo que ocurrió entre los profesores y la directora del colegio público, y de lo que no sucedió en el caso del colegio particular en el que las faltas de respeto que se generaron entre un grupo de profesores y el jefe de UTP se relacionaron con una baja evaluación de la estima personal. Entre integridad y estima personal también hay una estrecha relación, ya que un profesional que es capaz de privilegiar los intereses colectivos por sobre los individuales generará actuaciones a favor del bienestar del colectivo, como lo fue el caso de la directora del colegio público. La presencia que tuvo cada uno de estos criterios en el discurso de los actores fue distinta en cada escuela. Mientras en la municipal el más presente en los discursos de los profesionales fue el criterio de competencia profesional, en el particular fue el de integridad y estima personal. En ambos casos, el criterio menos tematizado fue el del respeto, probablemente porque era el mejor logrado en sus propias realidades, a excepción de la relación entre docentes y jefe de UTP del establecimiento particular. En síntesis, los criterios de discernimiento que conforman el nivel intrapersonal de la confianza revelaron la existencia de un ambiente poco propicio para el desarrollo de la confianza en la escuela particular y más auspicioso para el caso de la municipal. 
La diferencia de puntaje entre ambos casos en el Índice de confianza y el comportamiento de los criterios de discernimiento permiten concluir que la forma en la que se ejerce el liderazgo educativo es quizá uno de los criterios que mayor peso tienen al momento de construir confianza en las relaciones interpersonales, tanto a nivel de relaciones entre profesores como a nivel de la relación entre director y docentes.

Al ser un estudio de caso con enfoque cualitativo, la principal limitación se encuentra en la imposibilidad de generalizar los resultados debido al tamaño de la muestra, compuesta por sólo dos escuelas.

\section{Referencias bibliográficas}

Barber, B. (1983). The logic and limits of trust. New Jersey: Rutgers University Press.

Bryk, A. \& Schneider, B. (2002). Trust in schools: a core resource for improvement. New York: Russell Sage Foundation.

Cole, R. L. (1973). Toward a model of political trust. American Journal of Political Science, 17, 809-17.

Coleman, J. (1988) Social Capital in the Creation of Human Capital. American Journal of Sociology, Vol. 94, Supplement: Organizations and Institutions: Sociological and Economic Approaches to the Analysis of Social Structure, S95-S120.

Cook, K., Hardin, R. \& Levi, M. (2005). Cooperation without trust? New York: Russell Sage Foundation.

Covey, S. y Merril, R. (2007). El factor confianza. El valor que lo cambia todo. Barcelona: Paidós.

Dar, O. (2010). Trust in co-workers and employee behaviours at work. International Review of Business Research Papers. Vol. 6, nº 1. February, 194-204.

Deutsch, M. (1958). Trust and suspicion. Journal of conflict resolution, 2, 265-79.

Ferres, N. \& Travaglione, T. (2003). The development and validation of the workplace trust survey (WTS): combining qualitative and quantitative methodologies. Paper to be presented at APROS, Mexico 2003 Emotions, Attitudes and Culture Stream.

Forsyth, P., Barnes, L. \& Adams, C. (2006). Trust-effectiveness patterns in schools. Journal of Educational Administration, 44 (2)122-141. Doi: 10.1108/09578230610652024

Fukuyama, F. (1996). "Confianza (Trust)". Las virtudes sociales y la capacidad de generar prosperidad. Buenos Aires: Editorial Atlántida.

Fundación Chile (2006). Perfiles de competencias directivas, docentes y profesionales de apoyo: competencias funcionales. Programa Educación, Gestión Escolar. Recuperado de http:// www.educarchile.cl/ech/pro/app/detalle?ID=117200

Gillespie, N. \& Mann, L. (2000). The building blocks of trust: the role of transformational leadership and shared values in predicting team members trust in their leaders. Paper presented at the 2000 Academy of Management Conference. 
Goddard, R. D., Tschannen-Moran, M. \& Hoy, W. K. (2001). A multilevel examination of the distribution and effects of teacher trust in students and parents in urban elementary schools. Elementary School Journal, 102 (1), 3-17.

Hardin, R. (2002). Distrust. New York: Russell Sage Foundation.

Harjinder, G., Boies, K., Finegan, J. \& McNally, J. (2005). Antecedents of trust: establishing a boundary condition for the relation between propensity to trust and intention to trust. Journal of business and psychology, Vol. 19 (3), 287-302. Doi: 10.1007/s10869-004-2229-8

Hoy, W. \& Henderson, J. (1983). Principal authenticity, school climate and pupil-control orientation. Alberta journal of educational research. 29, 123-130.

Hoy, W. \& Tschannen-Moran, M. (1999). Five faces of trust: an empirical confirmation in urban elementary schools. Journal of school leadership, Vol. 9 (3), 184-208.

Kramer, R. M. \& Tyler, T. R. (1996). Trust in organizations: Frontiers of theory and research. California: Sage Publications.

Kramer, R. (1999). Trust and distrust in organizations: emerging perspectives, enduring questions. Annual Review of Psychology, Vol. 50 (1), 569-598. Doi: 10.1146/annurev. psych.50.1.569

Lewicki, R., McAllister, D. \& Bies, R. (1998). Trust and distrust: new relationships and realities. Academy of management review, Vol. 23(3), 438-458.

Lipman, M. (1990). Investigación social. Madrid: Ediciones de la Torre.

Luhmann, N. (1996). Confianza. Barcelona: Anthropos.

Mayer, R., J. \& Schoorman, D. (1995). An integrative model of organizational trust. The academy of management review, Vol. 20 (3), 709-734. Doi: 10.5465/AMR.1995.9508080335

Misztal, B. (1996). Trust in modern societies: the search for the bases of social order. Cambridge Mass: Polity Press.

Park, S., Henkin, A. \& Egley, R. (2005). Teacher team commitment, teamwork and trust: exploring associations. Journal of Educational Administration, 43 (5), 462-479.

Doi: 10.1108/09578230510615233

Parra, C. (2001). Autoridad y confianza: dos caras de la misma moneda. Educación y educadores, Vol. 4, 21-32.

Putnam, R. (2000). Bowling Alone. The Collapse and revival of American community. New York: Simon \& Schuster.

Putnam, R. (1995). Bowling Alone: America's Declining Social Capital. Journal of Democracy. 6:1, Jan 1995, 65-78. 
Saunders, M. \& Thornhill, A. (2004). Trust and distrust in organizations: an exploration using an organizational justice framework. European Journal of Work and Organizational Psychology, vol. 13 (4), 493-515. Doi: 10.1080/13594320444000182

Shalins, M. (1972). Stone Age Economics. Chicago: Aldine-Atherton.

Sztompka, P. (1999). Trust: a sociological theory. Cambridge: Cambridge University Press.

Tapia, C., Becerra, S., Mansilla, J. \& Saavedra, J. (2011). Liderazgo de los directivos docentes en contextos vulnerables. Educación y educadores, Vol. 14 (2), 389-409.

Tschannen-Moran, M. \& Hoy, W. K. (2000). A multidisciplinary analysis of the nature, meaning, and measurement of trust. Review of Educational Research, 71, 547-593.

Tschannen-Moran, M. \& Hoy, W. K. (1998). Trust in schools: A conceptual and empirical analysis. Journal of Educational Administration, 36, 334-352

Uline, C., Miller, D. \& Tschannen-Moran, M. (1998). School effectiveness: The underlying dimensions. Educational Administration Quarterly, 34, 462-483.

Wicks, A., Berman, S. \& Jones, T. (1999). The structure of optimal trust: moral and strategic implications. The academy of management review, Vol. 24 (1), 99-116. Doi: 10.2307/259039

Wolfe, R. N. (1976). Trust, anomia, and the locus of control: alienation of US college students in 1964, 1969, 1974. Journal of Social Psychology, 199, 151-72. Doi: $\underline{10.1080 / 00224545.1976 .9711920}$

Zapata, C., Gómez, M. \& Rojas, M. C. (2010). Modelado de la relación de confianza profesorestudiante en la docencia universitaria. Educación y educadores. Vol. 13 (1), 77-90.

\section{(Endnotes)}

${ }^{1}$ La dirección del enlace es: http://mxtsch.people.wm.edu/research_tools.php

2 Si bien los padres, apoderados y estudiantes no fueron el foco de la investigación, dado que estaban incluidos en el instrumento se optó por incorporarlos al análisis de modo de no subutilizar las potencialidades del cuestionario.

${ }^{3}$ Disponibles en el siguiente enlace: http://mxtsch.people.wm.edu/research_tools.php

${ }^{4}$ Consiste en la Subvención Escolar Preferencial (SEP) destinada al mejoramiento de la calidad de la educación de los establecimientos educacionales subvencionados. 